Editorial

\title{
Acknowledgement to Reviewers of Journal of Functional Biomaterials in 2019
}

Journal of Functional Biomaterials Editorial Office

MDPI, St. Alban-Anlage 66, 4052 Basel, Switzerland

Published: 20 January 2020

The editorial team greatly appreciates the reviewers who have dedicated their considerable time and expertise to the journal's rigorous editorial process over the past 12 months, regardless of whether the papers are finally published or not. In 2019, a total of 56 papers were published in the journal, with a median time to first decision of 18 days and a median time from submission to publication of 44 days. The editors would like to express their sincere gratitude to the following reviewers for their generous contribution in 2019:

Aguilar, Ludwig Erik

Alvarez-Lorenzo, Carmen

Ambu, Rita

Amza, Catalin

Baino, Francesco

Banas, Jeffrey

Banchelli, Martina

Barbeck, Mike

Beltrán, Ana M.

Bernardi, Sara

Boda, Sunil Kumar

Bouropoulos, Nikolaos

Boyer, Christen J.

Bruzell, Ellen

Cacciotti, Ilaria

Casimiro, Maria Helena

Chateigner, Daniel

Chen, Zhitong

Chereddy, Kiran

Chern, Edward

Chin, Kok Yong

Ciapetti, Gabriela

Crisci, Alessandro

Csapo, Edit

Daprile, Giuseppe

Diaz-Rodriguez, Patricia

Dinca, Ana

Dodero, Veronica

Donnermeyer, David

Dorozhkin, Sergey V.

Dutour Sikirić, Maja

Eglin, David

Elder, Steve
Fernández-Arévalo, Mercedes

Figueiras, Ana

Fregnan, Federica

Fröhlich, Eleonore

Gabrić, Dragana

Garcia-Gonzales, Carlos

Giudice, Giuseppe Lo

Gomez-Lazaro, Maria

Grzech-Leśniak, Kinga

Gu, Chunju

Gundapaneni, Dinesh

Hama, Susumu

Hamon, Morgan

He, Jingwei

Hegedűs, Csaba

Hixon, Katherine R.

Hoshiba, Takashi

Hsieh, Chih-Chen

$\mathrm{Hu}$, Yang

Huang, Yugang

Ibrahim, Toni

Illescas Montes, Rebeca Illescas

Incarnato, Loredana

Iordache, Florin

Iseki, Sachiko

Jammalamadaka, Udayabhanu

Joly-Duhamel, Christine

Kazek-Kęsik, Alicja

Keller, Brandis

Kengelbach-Weigand, Annika

Kevadiya, Bhavesh

Kijenska, Ewa

Kolmas, Joanna 
Koltz, Michael T.

Koutavarapu, Ravindranadh

Kurečič, Manja

Lee, Miyoung

Li, Ming-Chia

Lin, Maohua

Lin, Tz-Feng

Lindberg, Gabriella

Magyari, Klara

Majumder, Poulami

Marrazzo, Pasquale

Marto, Carlos Miguel

Meisel, Hans-Jörg

Messias, Ana

Metzinger, Laurent

Mokhtari, Sahar

Mukherjee, Sudip

Mulloy, Barbara

Muntimadugu, Eameema

Nam, Seung Yun

Nandi, Saikat

Narayanan, Ganesh

$\mathrm{Ng}$, Wei Long

Nguyen, Ba Thuy Linh

Nicholson, John W.

Nisnevitch, Marina

Okunkova, Anna A.

Otsuka, Yuta

Padmanabhan, Jagannath

Pagano, Stefano

Paknahad, Ali

Patterson, Jennifer

Peng, Chao

Perale, Giuseppe

Piluso, Susanna

Pogorielov, Maksym

Rabadán-Ros, Rubén

Raspanti, Mario

Ríos-Carrasco, Blanca
Rizzolio, Flavio

Rodríguez-Lorenzo, Luis

Rodríguez-Lozano, Francisco Javier

Román-Doval, Ramón

Sapudom, Jiranuwat

Scarano, Antonio

Schmidt, Franziska

Sefat, Farshid

Shen, Guofang

Shojaeiarani, Jamileh

Sills, E Scott

Solitro, Giovanni

Strudwick, Xanthe

Sutariya, Vijaykumar

Tabata, Yasuhiko

Tallarico, Marco

Tang, Houliang

Ting, Jeffrey M.

Tölli, Hanna

Truong, Vi Khanh

Tsai, Ang-Chen

Tsuchiya, Akira

Turco, Gianluca

Vilà, Anna

Walsh, Laurence J.

Walsh, Pamela J.

Wang, Xiaoju

Wang, Yuchen

Welshhans, Kristy

Witek, Lukasz

Yadavalli, Nataraja Sekhar

Yang, Tao

Yazdi, Iman

$\mathrm{Yu}, \mathrm{Lu}$

Zeitani, Jacob

Zhang, Yi

Zhou, Yubin

Zhu, Li

(C) 2020 by the author. Licensee MDPI, Basel, Switzerland. This article is an open access article distributed under the terms and conditions of the Creative Commons Attribution (CC BY) license (http://creativecommons.org/licenses/by/4.0/). 